Terue Okamura

Saori Tanaka

Koichi Koyama

Norifumi Nishida

Hideo Daikokuya

Toshiyuki Matsuoka

Kenji Kishimoto

Masakatsu Hatagawa

Hiroaki Kudoh

Ryusaku Yamada

Published online: 27 February 2002

(C) Springer-Verlag 2002

The online version of the original article can be found at http://dx.doi.org/10.1007/ s00330-001-1283-0

T. Okamura ( $) \cdot S$. Tanaka $\cdot$ K. Koyama

N. Nishida $\cdot$ H. Daikokuya $\cdot$ T. Matsuoka

R. Yamada

Department of Radiology,

Osaka City University School of Medicine,

1-4-3, Ashahi-machi, Abeno-ku,

Osaka 545-8585, Japan

e-mail: tokamura@msic.med.osaka-cu.ac.jp

Tel.: +81-6-66453831

Fax: +81-6-66466655

K. Kishimoto $\cdot$ M. Hatagawa $\cdot$ H. Kudoh Department of Radiology,

Osaka City University Hospital,

1-5-7, Ashahi-machi, Abeno-ku,

Osaka 545-8586, Japan

\section{Clinical evaluation of digital radiography based on a large-area cesium iodide-amorphous silicon flat-panel detector compared with screen-film radiography for skeletal system and abdomen}

\section{Eur Radiol (2002) \\ DOI 10.1007/s00330-001-1283-0}

Please note that the fourth author's proper name is as follows: Norifumi Nishida. 